\title{
Correlation between oxygen isotope effects on transition temperature and magnetic penetration depth in high-temperature superconductors close to optimal doping
}

\author{
R. Khasanov, ${ }^{1,2}$ A. Shengelaya, ${ }^{3}$ K. Conder ${ }^{4}$ E. Morenzoni, ${ }^{2}$ I. M. Savić, ${ }^{5}$ J. Karpinski, ${ }^{6}$ and H. Keller ${ }^{1}$ \\ ${ }^{1}$ Physik-Institut der Universität Zürich, Winterthurerstrasse 190, CH-8057 Zürich, Switzerland \\ ${ }^{2}$ Laboratory for Muon Spin Spectroscopy, Paul Scherrer Institut, CH-5232 Villigen PSI, Switzerland \\ ${ }^{3}$ Physics Institute of Tbilisi State University, Chavchavadze 3, GE-0128 Tbilisi, Georgia \\ ${ }^{4}$ Laboratory for Developments and Methods, Paul Scherrer Institut, CH-5232 Villigen PSI, Switzerland \\ ${ }^{5}$ Faculty of Physics, University of Belgrade, 11001 Belgrade, Serbia and Montenegro \\ ${ }^{6}$ Solid State Physics Laboratory, ETH 8093 Zürich, Switzerland
}

(Received 12 April 2006; revised manuscript received 6 July 2006; published 9 August 2006)

The oxygen-isotope $\left({ }^{16} \mathrm{O}-{ }^{18} \mathrm{O}\right)$ effect $(\mathrm{OIE})$ on the in-plane magnetic penetration depth $\lambda_{a b}(0)$ in optimally doped $\mathrm{YBa}_{2} \mathrm{Cu}_{3} \mathrm{O}_{7-\delta}$ and $\mathrm{La}_{1.85} \mathrm{Sr}_{0.15} \mathrm{CuO}_{4}$, and in slightly underdoped $\mathrm{YBa}_{2} \mathrm{Cu}_{4} \mathrm{O}_{8}$ and $\mathrm{Y}_{0.8} \mathrm{Pr}_{0.2} \mathrm{Ba}_{2} \mathrm{Cu}_{3} \mathrm{O}_{7-\delta}$ was studied by means of muon-spin rotation. A substantial OIE on $\lambda_{a b}(0)$ with an OIE exponent $\beta_{\mathrm{O}}$ $=-d \ln \lambda_{a b}(0) / d \ln M_{\mathrm{O}} \approx-0.2\left(M_{\mathrm{O}}\right.$ is the mass of the oxygen isotope), and a small OIE on the transition temperature $T_{c}$ with an OIE exponent $\alpha_{\mathrm{O}}=-d \ln T_{c} / d \ln M_{\mathrm{O}} \simeq 0.02-0.1$ were observed. The observation of a substantial isotope effect on $\lambda_{a b}(0)$, even in cuprates where the OIE on $T_{c}$ is small, indicates that lattice effects play an important role in cuprate high-temperature superconductors.

DOI: 10.1103/PhysRevB.74.064504 PACS number(s): 74.72. $-\mathrm{h}$, 76.75.+i, 82.20.Tr, 74.25.Kc

\section{INTRODUCTION}

The observation of unusual isotope effects in cuprate high-temperature superconductors (HTSs) on the transition temperature $T_{c},{ }^{1-4}$ and on the zero-temperature in-plane magnetic penetration depth $\lambda_{a b}(0),{ }^{5-15}$ poses a challenge to the understanding of high-temperature superconductivity. To date, most isotope effect studies on $T_{c}$ and $\lambda_{a b}(0)$ in HTSs were performed by substituting oxygen ${ }^{16} \mathrm{O}$ with ${ }^{18} \mathrm{O}$. It was observed that the oxygen isotope $\left({ }^{16} \mathrm{O}-{ }^{18} \mathrm{O}\right)$ effect (OIE) on both $T_{c}$ and $\lambda_{a b}(0)$ have a tendency to increase with decreasing doping. ${ }^{5-15}$ Later on it was shown that for different families of HTS cuprates there is a universal correlation between the isotope shifts of these two quantities; ${ }^{9,12-15}$ namely, in the underdoped region $\Delta T_{c} / T_{c}$ and $\Delta \lambda_{a b}(0) / \lambda_{a b}(0)$ scale linearly with respect to each other with $\left|\Delta T_{c} / T_{c}\right| \simeq\left|\Delta \lambda_{a b}(0) / \lambda_{a b}(0)\right|$. However, close to optimal doping the situation is not so clear. Khasanov and co-workers ${ }^{11,12}$ observed that in optimally doped $\mathrm{YBa}_{2} \mathrm{Cu}_{3} \mathrm{O}_{7-\delta}$ the small OIE on $T_{c}$ is associated with a rather big isotope shift of $\lambda_{a b}$ that is even compatible with the OIE on $\lambda_{a b}$ in underdoped cuprates. In contrast, Tallon et al. ${ }^{15}$ showed that in slightly overdoped $\mathrm{La}_{2-x} \mathrm{Sr}_{x} \mathrm{Cu}_{1-y} \mathrm{Zn}_{y} \mathrm{O}_{4}$ the OIE on $\lambda_{a b}(0)$ is zero while the OIE on $T_{c}$ still remains substantial.

In this paper we concentrate on studies of the OIE on $T_{c}$ and $\lambda_{a b}(0)$ in optimally doped $\mathrm{La}_{1.85} \mathrm{Sr}_{0.15} \mathrm{CuO}_{4}$ and $\mathrm{YBa}_{2} \mathrm{CuO}_{7-\delta}$, as well as in slightly underdoped $\mathrm{YBa}_{2} \mathrm{Cu}_{4} \mathrm{O}_{8}$ and $\mathrm{Y}_{0.8} \mathrm{Pr}_{0.2} \mathrm{Ba}_{2} \mathrm{CuO}_{7-\delta}$. All the samples show a rather small OIE on $T_{c}$ associated with a relatively large OIE on $\lambda_{a b}(0)$. The oxygen isotope exponents on $T_{c}\left(\alpha_{\mathrm{O}}=-d \ln T_{c} / d \ln M_{\mathrm{O}}\right.$, where $M_{\mathrm{O}}$ is the mass of the oxygen isotope) and the in-plane magnetic penetration depth $\lambda_{a b}(0) \quad\left(\beta_{\mathrm{O}}\right.$ $\left.=-d \ln \lambda_{a b}(0) / d \ln M_{\mathrm{O}}\right)$ were found to be $\alpha_{\mathrm{O}}=0.024(8)$ and $\beta_{\mathrm{O}}=-0.21(4)$ for $\mathrm{YBa}_{2} \mathrm{CuO}_{7-\delta}, \alpha_{\mathrm{O}}=0.10(1)$ and $\beta_{\mathrm{O}}$ $=-0.19(6)$ for $\mathrm{Y}_{0.8} \mathrm{Pr}_{0.2} \mathrm{Ba}_{2} \mathrm{CuO}_{7-\delta}, \alpha_{\mathrm{O}}=0.048(8)$ and $\beta_{\mathrm{O}}$ $=-0.18(6)$ for $\mathrm{YBa}_{2} \mathrm{Cu}_{4} \mathrm{Cu}_{4} \mathrm{O}_{8}$, and $\alpha_{\mathrm{O}}=0.08(1)$ and $\beta_{\mathrm{O}}$ $=-0.18(5)$ for $\mathrm{La}_{1.85} \mathrm{Sr}_{0.15} \mathrm{CuO}_{4}$. The fact that a substantial
OIE on $\lambda_{a b}(0)$ is observed even in cuprates having a relatively small OIE on $T_{c}$ suggests that lattice effects have to be considered in any realistic model of high-temperature superconductivity.

\section{EXPERIMENTAL DETAILS}

Powder samples of $\mathrm{Y}_{1-x} \mathrm{Pr}_{x} \mathrm{Ba}_{2} \mathrm{Cu}_{3} \mathrm{O}_{7-\delta}, \mathrm{YBa}_{2} \mathrm{Cu}_{4} \mathrm{O}_{8}$, and $\mathrm{La}_{1.85} \mathrm{Sr}_{0.15} \mathrm{CuO}_{4}$ were synthesized by solid state reactions. ${ }^{16,17}$ Oxygen isotope exchange was performed while heating the samples in ${ }^{18} \mathrm{O}_{2}$ gas. In order to ensure that the ${ }^{16} \mathrm{O}$ - and ${ }^{18} \mathrm{O}$-substituted samples are subjects of the same thermal history, the annealing of the two samples is performed simultaneously in ${ }^{16} \mathrm{O}_{2}$ and ${ }^{18} \mathrm{O}_{2}$ (95\% enriched) gas, respectively. The ${ }^{18} \mathrm{O}$ content of the samples, as determined from a change of the sample weight after the isotope exchange, was found to be $90 \%$ for $\mathrm{YBa}_{2} \mathrm{Cu}_{3} \mathrm{O}_{7-\delta}, 82 \%$ for $\mathrm{Y}_{0.8} \mathrm{Pr}_{0.2} \mathrm{Ba}_{2} \mathrm{Cu}_{3} \mathrm{O}_{7-\delta}$ and $\mathrm{YBa}_{2} \mathrm{Cu}_{4} \mathrm{O}_{8}$, and $85 \%$ for $\mathrm{La}_{1.85} \mathrm{Sr}_{0.15} \mathrm{CuO}_{4}$. The total oxygen content for the $\mathrm{YBa}_{2} \mathrm{Cu}_{3} \mathrm{O}_{7-\delta}$ and $\mathrm{La}_{1.85} \mathrm{Sr}_{0.15} \mathrm{CuO}_{4}$ samples was determined by means of very accurate volumetric analysis. ${ }^{16}$ The oxygen contents are 6.951(2) [6.953(2)] and 3.9981(3) [3.9976(3)] for the ${ }^{16} \mathrm{O}-\left({ }^{18} \mathrm{O}\right)$ substituted $\mathrm{YBa}_{2} \mathrm{Cu}_{3} \mathrm{O}_{7-\delta}$ and $\mathrm{La}_{1.85} \mathrm{Sr}_{0.15} \mathrm{CuO}_{4}$ samples, respectively.

In order to determine the OIE on $T_{c}$, field-cooled magnetization $\left(M_{F C}\right)$ measurements were performed with a superconducing quantum interference design magnetometer in a field of $1 \mathrm{mT}$ at temperatures between 1.75 and $100 \mathrm{~K}$. For the investigation of the OIE on $\lambda_{a b}(0)$, transverse-field muon-spin resonance $(\mu \mathrm{SR})$ experiments were performed at the Paul Scherrer Institute (PSI), Switzerland, using the $\pi$ M3 $\mu$ SR facility. The samples were field cooled from far above $T_{c}$ in a field of $0.2 \mathrm{~T}$. In a powder sample the magnetic penetration depth $\lambda$ can be extracted from the muon-spin depolarization rate $\sigma(T) \propto 1 / \lambda^{2}(T)$, which probes the second moment $\left\langle\Delta B^{2}\right\rangle^{1 / 2}$ of the probability distribution of the local 

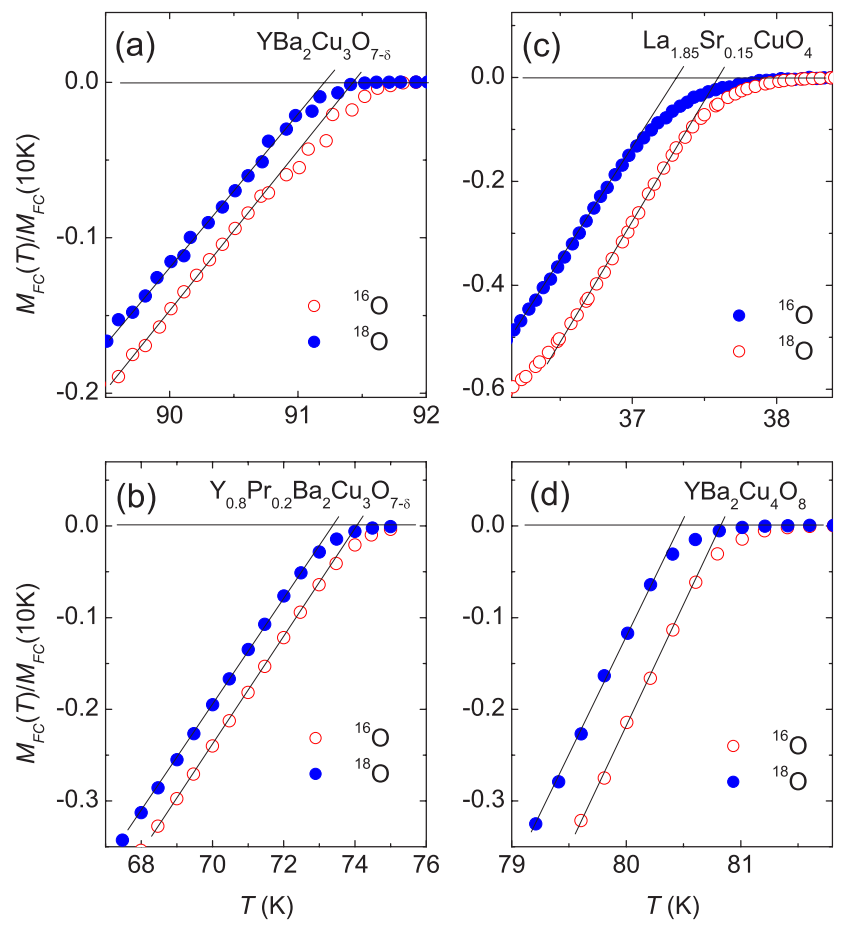

FIG. 1. (Color online) Section near $T_{c}$ of the low-field (1 $\mathrm{mT}$, field cooled) magnetization curves (normalized to the value at $10 \mathrm{~K}$ ) for ${ }^{16} \mathrm{O}$ and ${ }^{18} \mathrm{O}$-substituted $\mathrm{YBa}_{2} \mathrm{Cu}_{3} \mathrm{O}_{7-\delta} \quad$ (a), $\mathrm{Y}_{0.8} \mathrm{Pr}_{0.2} \mathrm{Ba}_{2} \mathrm{Cu}_{3} \mathrm{O}_{7-\delta}$ (b), $\mathrm{La}_{1.85} \mathrm{Sr}_{0.15} \mathrm{CuO}_{4}$ (c), and $\mathrm{YBa}_{2} \mathrm{Cu}_{4} \mathrm{O}_{8}$ (d).

magnetic field function $p(B)$ in the mixed state. ${ }^{18}$ For highly anisotropic layered superconductors (like cuprate superconductors) $\lambda$ is mainly determined by the in-plane penetration depth $\lambda_{a b} \cdot{ }^{18} \sigma(T) \propto 1 / \lambda_{a b}^{2}(T)$. The depolarization rate $\sigma$ was extracted from the $\mu$ SR time spectra using a Gaussian relaxation function $R(t)=\exp \left(-\sigma^{2} t^{2} / 2\right)$. The superconducting contribution $\sigma_{s c}$ was obtained by subtracting the dipolar contribution $\sigma_{n m}$ measured above $T_{c}$ as $\sigma_{s c}^{2}=\sigma^{2}-\sigma_{n m}^{2}$.

\section{RESULTS AND DISCUSSION}

Figure 1 shows the temperature dependences of the fieldcooled magnetization for the ${ }^{16} \mathrm{O}-\left({ }^{18} \mathrm{O}\right)$ substituted samples investigated in this work. It is seen that the magnetization curves for the ${ }^{18} \mathrm{O}$-substituted samples are shifted almost parallel to lower temperatures, implying that the $T_{c}$ 's of the ${ }^{18} \mathrm{O}$ samples are lower than those of the ${ }^{16} \mathrm{O}$ samples.

The results of the OIE on $T_{c}$ are summarized in Table I. The transition temperature $T_{c}$ was determined as the temperature where the linearly extrapolated transition slope intersects the zero line. The OIE exponent $\alpha_{\mathrm{O}}$ is defined by $\alpha_{\mathrm{O}}=-d \ln T_{c} / d \ln M_{\mathrm{O}}$. Taking into account an incomplete oxygen isotope exchange $\left(90 \%\right.$ for $\mathrm{YBa}_{2} \mathrm{Cu}_{3} \mathrm{O}_{7-\delta}, 82 \%$ for $\mathrm{Y}_{0.8} \mathrm{Pr}_{0.2} \mathrm{Ba}_{2} \mathrm{Cu}_{3} \mathrm{O}_{7-\delta}$ and $\mathrm{YBa}_{2} \mathrm{Cu}_{4} \mathrm{O}_{8}$, and $85 \%$ for $\mathrm{La}_{1.85} \mathrm{Sr}_{0.15} \mathrm{CuO}_{4}$ ), we found $\alpha_{\mathrm{O}}=0.024(8), 0.10(1), 0.048(8)$, and $0.08(1)$ for $\mathrm{YBa}_{2} \mathrm{Cu}_{3} \mathrm{O}_{7-\delta}, \quad \mathrm{Y}_{0.8} \mathrm{Pr}_{0.8} \mathrm{Ba}_{2} \mathrm{Cu}_{3} \mathrm{O}_{7-\delta}$, $\mathrm{YBa}_{2} \mathrm{Cu}_{4} \mathrm{O}_{8}$, and $\mathrm{La}_{1.85} \mathrm{Sr}_{0.15} \mathrm{CuO}_{4}$, respectively. Note that these values are in fair agreement with the previously published results. $^{2-15}$
TABLE I. Summary of the OIE results on $T_{c}$ for $\mathrm{Y}_{1-x} \mathrm{Pr}_{x} \mathrm{Ba}_{2} \mathrm{Cu}_{3} \mathrm{O}_{7-\delta}(x=0.0,0.2), \mathrm{YBa}_{2} \mathrm{C}_{4} \mathrm{O}_{8}$, and $\mathrm{La}_{1.85} \mathrm{Sr}_{0.15} \mathrm{CuO}_{4}$. The values of $\Delta T_{c} / T_{c}$ and $\alpha_{\mathrm{O}}$ are corrected for the incomplete ${ }^{18} \mathrm{O}$ exchange (see text for an explanation).

\begin{tabular}{ccccc}
\hline \hline & $\begin{array}{c}{ }^{16} \mathrm{O} \\
{ }^{18} \mathrm{O}\end{array}$ & & \\
& $T_{c}$ & $T_{c}$ & $\Delta T_{c} / T_{c}$ & \\
Sample & $(\mathrm{K})$ & $(\mathrm{K})$ & $(\%)$ & $\alpha_{\mathrm{O}}$ \\
\hline $\mathrm{YBa}_{2} \mathrm{Cu}_{3} \mathrm{O}_{7-\delta}$ & $91.45(5)$ & $91.20(5)$ & $-0.3(1)$ & $0.024(8)$ \\
$\mathrm{Y}_{0.8} \mathrm{Pr}_{0.2} \mathrm{Ba}_{2} \mathrm{Cu}_{3} \mathrm{O}_{7-\delta}$ & $74.0(1)$ & $73.2(1)$ & $-1.3(3)$ & $0.104(12)$ \\
$\mathrm{YBa}_{2} \mathrm{Cu}_{4} \mathrm{O}_{8}$ & $80.86(5)$ & $80.46(5)$ & $-0.6(1)$ & $0.048(8)$ \\
$\mathrm{La}_{1.85} \mathrm{Sr}_{0.15} \mathrm{CuO}_{4}$ & $37.63 .(2)$ & $37.31(2)$ & $-1.0(1)$ & $0.08(1)$ \\
\hline \hline
\end{tabular}

Figure 2 shows the temperature dependences of the superconducting part of the $\mu \mathrm{SR}$ depolarization rate $\sigma_{s c} \propto \lambda_{a b}^{-2}$ of the samples studied. It is seen that the data points for the ${ }^{16} \mathrm{O}$-substituted samples are systematically higher than those for the ${ }^{18} \mathrm{O}$ ones, implying that an oxygen isotope shift on $\sigma_{s c}$ is present. As in Ref. 18, the data in Fig. 2 were fitted to the power law $\sigma_{s c}(T) / \sigma_{s c}(0)=1-\left(T / T_{c}\right)^{n}$ with $\sigma_{s c}(0)$ and $n$ as free parameters, and $T_{c}$ taken from the magnetization measurements (see Table I). The values of $\sigma_{s c}(0)$ obtained from the fits are listed in Table II and are in agreement with previous results. ${ }^{18-20}$ The exponents $n$ were found to be the same within error for each set of ${ }^{16} \mathrm{O}-{ }^{18} \mathrm{O}$ samples, implying that $\sigma_{s c}$ has nearly the same temperature dependence for the two isotopes (see Fig. 2). The values of the relative shift of $\lambda_{a b}(0)$ and the oxygen isotope exponent $\beta_{\mathrm{O}}$ obtained from the measured values of $\sigma_{s c}(0)$ and corrected for the incomplete ${ }^{18} \mathrm{O}$ exchange are summarized in Table II.

Note that, the observed OIE's on $T_{c}$ and $\lambda_{a b}(0)$ are not caused by a possible difference in the carrier concentrations

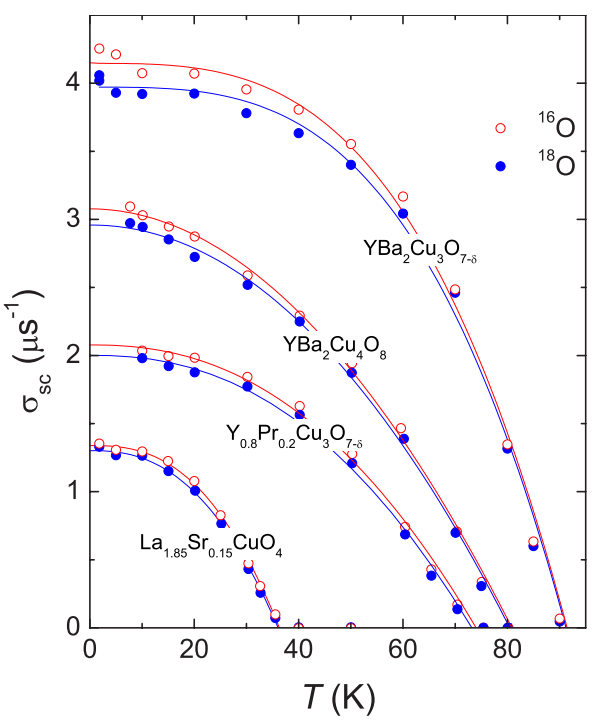

FIG. 2. (Color online) Temperature dependences of depolarization rate $\sigma_{s c}$ for ${ }^{16} \mathrm{O}$ - and ${ }^{18} \mathrm{O}$-substituted (from the top to the bottom) $\quad \mathrm{YBa}_{2} \mathrm{Cu}_{3} \mathrm{O}_{7-\delta}, \quad \mathrm{YBa}_{2} \mathrm{Cu}_{4} \mathrm{O}_{8}, \quad \mathrm{Y}_{0.8} \mathrm{Pr}_{0.2} \mathrm{Ba}_{2} \mathrm{Cu}_{3} \mathrm{O}_{7-\delta}$, and $\mathrm{La}_{1.85} \mathrm{Sr}_{0.15} \mathrm{CuO}_{4}$ samples (200 $\mathrm{mT}$, field cooled). The solid lines correspond to fits to the power law $\sigma_{s c}(T) / \sigma_{s c}(0)=1-\left(T / T_{c}\right)^{n}$. The error bars are smaller than the size of the points. 
TABLE II. Summary of the OIE results on $\lambda_{a b}(0)$ for $\mathrm{Y}_{1-x} \mathrm{Pr}_{x} \mathrm{Ba}_{2} \mathrm{Cu}_{3} \mathrm{O}_{7-\delta} \quad(x=0.0,0.2), \quad \mathrm{YBa}_{2} \mathrm{Cu}_{4} \mathrm{O}_{8}, \quad$ and $\mathrm{La}_{1.85} \mathrm{Sr}_{0.15} \mathrm{CuO}_{4}$. The values of $\Delta \lambda_{a b}(0) / \lambda_{a b}(0)$ and $\beta_{\mathrm{O}}$ are corrected for the incomplete ${ }^{18} \mathrm{O}$ exchange (see text for an explanation).

\begin{tabular}{ccccc}
\hline \hline Sample & $\begin{array}{c}{ }^{16} \mathrm{O} \\
\sigma(0) \\
\left(\mu \mathrm{s}^{-1}\right)\end{array}$ & $\begin{array}{c}{ }^{18} \mathrm{O} \\
\sigma(0) \\
\left(\mu \mathrm{s}^{-1}\right)\end{array}$ & $\begin{array}{c}\Delta \lambda_{a b}(0) / \lambda_{a b}(0) \\
(\%)\end{array}$ & $\beta_{\mathrm{O}}$ \\
\hline $\mathrm{YBa}_{2} \mathrm{Cu}_{3} \mathrm{O}_{7-\delta}$ & $4.15(4)$ & $3.96(4)$ & $2.6(5)$ & $-0.21(4)$ \\
$\mathrm{Y}_{0.8} \mathrm{Pr}_{0.2} \mathrm{Ba}_{2} \mathrm{Cu}_{3} \mathrm{O}_{7-\delta}$ & $2.08(1)$ & $2.00(1)$ & $2.4(7)$ & $-0.19(6)$ \\
$\mathrm{YBa}_{2} \mathrm{Cu}_{4} \mathrm{O}_{8}$ & $3.07(3)$ & $2.96(3)$ & $2.2(7)$ & $-0.18(6)$ \\
$\mathrm{La}_{1.85} \mathrm{Sr}_{0.15} \mathrm{CuO}_{4}$ & $1.34(1)$ & $1.29(1)$ & $2.2(6)$ & $-0.18(5)$ \\
\hline \hline
\end{tabular}

of the ${ }^{16} \mathrm{O}$ and ${ }^{18} \mathrm{O}$ samples. This is because the oxygen contents in the ${ }^{16} \mathrm{O}$ - and ${ }^{18} \mathrm{O}$-substituted $\mathrm{YBa}_{2} \mathrm{Cu}_{3} \mathrm{O}_{7-\delta}$ and $\mathrm{La}_{1.85} \mathrm{Sr}_{0.5} \mathrm{CuO}_{4}$ are the same within error (see Sec. II) and $\mathrm{YBa}_{2} \mathrm{Cu}_{4} \mathrm{O}_{8}$ is a stoichiometric compound with a fixed oxygen content. ${ }^{17,21}$ Additional arguments are given in Refs. $5-14$.

In order to demonstrate that the change of the oxygen content within the precision of our volumetric analysis cannot account for the observed OIE on $T_{c}$ and $\lambda_{a b}(0)$ we used the following procedure. Tallon et al. ${ }^{22}$ observed that in a wide range of doping $(0.05 \leqslant p \leqslant 0.19)$ the following empirical relation holds: $T_{c} \lambda_{a b}^{-2}(0) \propto p-0.05$ ( $p$ is the number of holes per planar $\mathrm{Cu}$ ). This implies that

$$
\frac{\Delta p}{p-0.05}=\frac{\Delta T_{c}}{T_{c}}-2 \frac{\Delta \lambda_{a b}(0)}{\lambda_{a b}(0)} .
$$

Taking into account that oxygen is divalent and that the unit cell of $\mathrm{YBa}_{2} \mathrm{Cu}_{3} \mathrm{O}_{7-\delta}$ contains two plane and one chain $\mathrm{Cu}$ atoms, the change in the hole concentration $\Delta p$ caused by the change of the oxygen content can be estimated as $\Delta p$ $=-2 \Delta y$ for $\mathrm{La}_{2-x} \mathrm{Sr}_{x} \mathrm{CuO}_{4-y}$ and $\Delta p=-(2 / 3) \Delta \delta$ for $\mathrm{YBa}_{2} \mathrm{Cu}_{3} \mathrm{O}_{7-\delta}$. For the above mentioned errors in the determination of the oxygen content $\left( \pm 0.002\right.$ for $\mathrm{YBa}_{2} \mathrm{Cu}_{3} \mathrm{O}_{7-\delta}$ and \pm 0.0003 for $\mathrm{La}_{1.85} \mathrm{Sr}_{0.15} \mathrm{CuO}_{4}$, see Sec. II) and with $p$ $\simeq 0.15$ for the optimally doped samples, one gets $\mid \Delta T_{c} / T_{c}$ $-2 \Delta \lambda_{a b}(0) / \lambda_{a b}(0) \mid<1.3 \%$ and $<0.6 \% \quad \mathrm{YBa}_{2} \mathrm{Cu}_{3} \mathrm{O}_{7-\delta}$ and $\mathrm{La}_{1.85} \mathrm{Sr}_{0.15} \mathrm{CuO}_{4}$, respectively. These values are more than five times smaller than one would obtain by substituting $\Delta T_{c} / T_{c}$ and $\Delta \lambda_{a b}(0) / \lambda_{a b}(0)$ in Eq. (1) by the values listed in Tables I and II.

In Fig. 3 the transition temperature $T_{c}$ is plotted versus the $\mu \mathrm{SR}$ depolarization rate $\sigma_{s c}(0) \propto \lambda_{a b}^{-2}(0)$ for the samples studied. Recent OIE results for $\mathrm{Y}_{x} \mathrm{Pr}_{1-x} \mathrm{Ba}_{2} \mathrm{Cu}_{3} \mathrm{O}_{7-\delta}$ (Refs. 9-11) and $\mathrm{La}_{2-x} \mathrm{Sr}_{x} \mathrm{CuO}_{4}$ (Refs. 6 and 8) are also included in the graph. Since the absolute values of $\lambda_{a b}(0)$ for the $\mathrm{La}_{2-x} \mathrm{Sr}_{x} \mathrm{CuO}_{4}$ samples studied in Refs. 6 and 8 are not known, the values of $\sigma_{s c}(0)$ for the ${ }^{16} \mathrm{O}$-substituted samples were estimated from comparison with previous data. ${ }^{23,24}$

According to Refs. 23 and 24 in the underdoped regime $T_{c}$ is proportional to $\sigma_{s c}(0) \propto \lambda_{a b}^{-2}(0)$ with a universal slope for most HTS families and saturates close to optimal doping to a value characteristic for each HTS family (Uemura rela- tion). Furthermore, recent experiments on an ultrathin $\mathrm{La}_{2-x} \mathrm{Sr}_{x} \mathrm{CuO}_{4}$ film clearly show that the Uemura relation $T_{c} \propto n_{s} / m^{*}$ holds when the superfluid density is modulated by an electric field. ${ }^{25}$ Superconductors that belong to the Y124 family (including $\mathrm{YBa}_{2} \mathrm{Cu}_{4} \mathrm{O}_{8}$ ) contain $\mathrm{CuO}$ chains free of disorder and thus exhibit enhanced values of $\lambda_{a b}^{-2}(0)$ compared to the Uemura line. ${ }^{26,27}$ The "universal" $T_{c}$ vs $\sigma_{s c}(0)$ curves for the HTS families $\mathrm{Y}_{x} \mathrm{Pr}_{1-x} \mathrm{Ba}_{2} \mathrm{Cu}_{3} \mathrm{O}_{7-\delta}$ (YPr123), $\mathrm{La}_{2-x} \mathrm{Sr}_{x} \mathrm{CuO}_{4}$ (La214), and $\mathrm{YBa}_{2} \mathrm{Cu}_{4} \mathrm{O}_{8}$ (Y124) are shown in Fig. 3. Figure 3 suggests that the relation between isotope shifts of $T_{c}$ and $\lambda_{a b}(0)$ can be explained qualitatively by the empirical Uemura line. Indeed $T_{c}$ and $\sigma_{s c}(0)$ for the ${ }^{18} \mathrm{O}$-substituted samples are always smaller than those for the ${ }^{16} \mathrm{O}$ samples. It is also seen that for samples close to optimal doping a small OIE on $T_{c}$ is associated with a rather large OIE on $\lambda_{a b}(0)$. In order to investigate these results in more detail, we plot in Fig. 4 the OIE shift of $\lambda_{a b}(0)$ $\left[\Delta \lambda_{a b}(0) / \lambda_{a b}(0)\right]$ versus the OIE shift of $T_{c}\left(\Delta T_{c} / T_{c}\right)$. It is remarkable that different experimental techniques [SQUID magnetization, ${ }^{6}$ magnetic torque, ${ }^{8}$ bulk $\mu \mathrm{SR},{ }^{9,10}$ low-energy (LE) $\mu \mathrm{SR}$ (Ref. 11)] and different types of samples (single crystals, ${ }^{8}$ powders,${ }^{6,9,10}$ thin films ${ }^{11}$ ) yield consistnt results within experimental error. However, one can easily see that the Uemura relation can explain the observed correlation between $\Delta \lambda_{a b}(0) / \lambda_{a b}(0)$ and $\Delta T_{c} / T_{c}$ only qualitatively but not quantitatively. Following Uemura et al. ${ }^{23,24}$ for different families of underdoped cuprates, $T_{c}$ scales linearly with the $\mu \mathrm{SR}$ depolarization rate $\sigma_{s c}(0) \propto \lambda_{a b}^{-2}(0)$, yielding $\Delta \lambda_{a b}(0) / \lambda_{a b}(0) \simeq 0.5\left|\Delta T_{c} / T_{c}\right|$ (line $A$ in Fig. 4). It is seen, however, that all the experimental points are systematically higher. At low doping level $\Delta \lambda_{a b}(0) / \lambda_{a b}(0) \simeq\left|\Delta T_{c} / T_{c}\right|$ (line

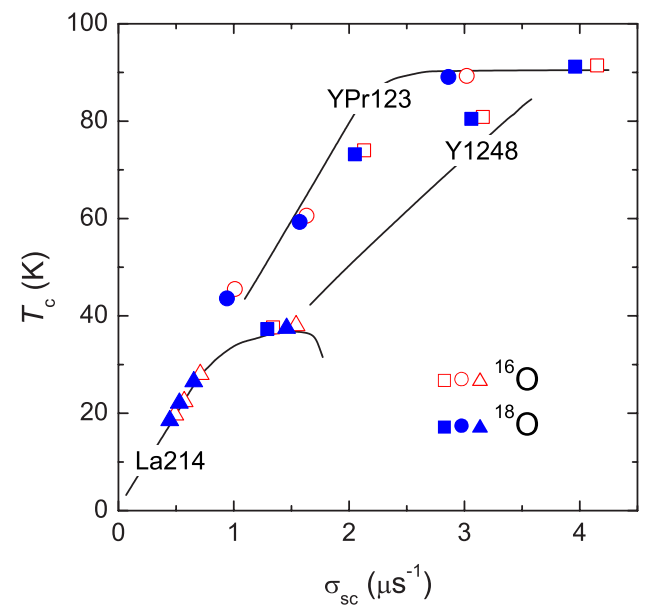

FIG. 3. (Color online) Plot of $T_{c}$ versus $\sigma_{s c}(0)$ for ${ }^{16} \mathrm{O}-$ (open symbols) and ${ }^{18} \mathrm{O}-$ (closed symbols) substituted $\mathrm{Y}_{1-x} \mathrm{Pr}_{x} \mathrm{Ba}_{2} \mathrm{Cu}_{3} \mathrm{O}_{7-\delta}, \mathrm{YBa}_{2} \mathrm{Cu}_{4} \mathrm{O}_{8}$, and $\mathrm{La}_{2-x} \mathrm{Sr}_{x} \mathrm{CuO}_{4}$. Squares are the $\mu \mathrm{SR}$ data obtained in the present study. Circles are bulk $\mu \mathrm{SR}$ data for $\mathrm{Y}_{1-x} \mathrm{Pr}_{x} \mathrm{Ba}_{2} \mathrm{Cu}_{3} \mathrm{O}_{7-\delta}$ (Refs. 9 and 10) and LE $\mu$ SR data for optimally doped $\mathrm{YBa}_{2} \mathrm{Cu}_{3} \mathrm{O}_{7-\delta}$ (Ref. 11). Triangles are torque magnetization and Meissner fraction data for $\mathrm{La}_{2-x} \mathrm{Sr}_{x} \mathrm{CuO}_{4}$ (Refs. 6 and $8)$. The solid lines correspond to the universal $T_{c}$ vs $\sigma_{s c}(0)$ relations for the YPr123, La214, and Y124 families of HTSs (Refs. 23, 24, 26, and 27). 
$B$ in Fig. 4), whereas close to the optimal doping $\Delta \lambda_{a b}(0) / \lambda_{a b}(0)$ is almost constant and considerably larger than $\Delta T_{c} / T_{c}\left[\Delta \lambda_{a b}(0) / \lambda_{a b}(0) \approx 10\left|\Delta T_{c} / T_{c}\right|\right] .^{12-14}$

According to the London theory $\lambda_{a b}^{-2}$ is proportional to the so-called superfluid density $\lambda_{a b}^{-2} \propto n_{s} / m_{a b}\left(n_{s}\right.$ is the density of supercarriers and $m_{a b}^{*}$ is the in-plane charge carrier mass). Concerning the relation between $\Delta \lambda_{a b}(0) / \lambda_{a b}(0)$ and $\Delta T_{c} / T_{c}$, one should distinguish two cases: (i) change of the carrier density $n$ by doping (note that $n$ is not necessarily equal to $n_{s}$ as discussed in Ref. 25), and (ii) change of the oxygen mass by isotope substitution. In the recent electrostatic modulation experiments, ${ }^{25}$ it was shown that the change of the carrier density within the same sample leads to $T_{c} \propto n_{s}$, and $\Delta \lambda_{a b}(0) / \lambda_{a b}(0)=0.5\left|\Delta T_{c} / T_{c}\right|$, in accordance with the Uemura relation in the underdoped regime. ${ }^{23,24}$ Note that in Ref. 25 carriers were implanted in or removed from the sample by changing the electric field, so the crystal lattice is not affected. This implies that in this case changes of both $T_{c}$ and $\lambda_{a b}(0)$ are due to a change of the carrier concentration $n_{s}$, while the in-plane charge carrier mass $m_{a b}^{*}$ stays constant. ${ }^{28}$ The isotope substitution, in contrast, modifies the lattice, but leaves the doping (oxygen content) unchanged (see discussion above). In addition, it is found that $\Delta \lambda_{a b}(0) / \lambda_{a b}(0)=\left|\Delta T_{c} / T_{c}\right|$, so there is a factor of 2 difference in the slope of lines $A$ and $B$ in Fig. 4. This factor of 2 can be explained by a simple model, assuming that $T_{c}$ $\propto n_{s}$ and $\lambda_{a b}^{-2}(0) \propto n_{s} / m_{a b}^{*}$ (London model). This implies that in case (i) described above, $\Delta \lambda_{a b}(0) / \lambda_{a b}(0)=0.5\left|\Delta T_{c} / T_{c}\right|$

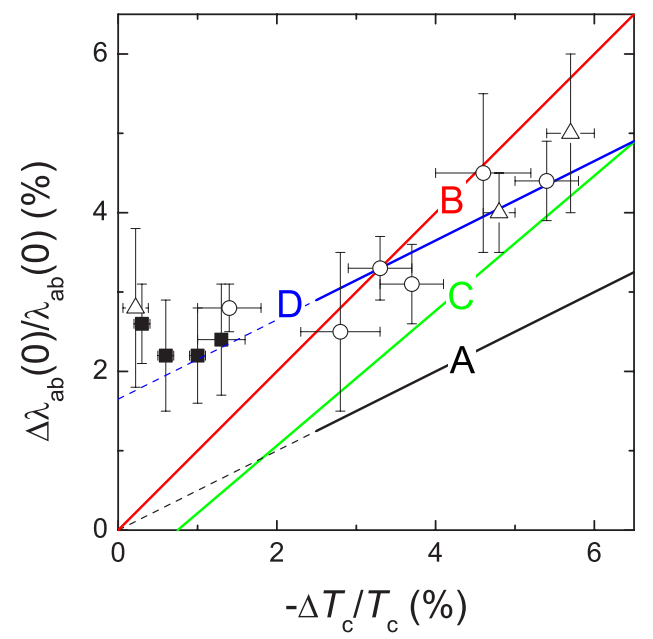

FIG. 4. (Color online) Plot of the OIE shift $\Delta \lambda_{a b}(0) / \lambda_{a b}(0)$ versus the OIE shift $-\Delta T_{c} / T_{c}$ for $\mathrm{Y}_{1-x} \mathrm{Pr}_{x} \mathrm{Ba}_{2} \mathrm{Cu}_{3} \mathrm{O}_{7-\delta}, \mathrm{YBa}_{2} \mathrm{Cu}_{4} \mathrm{O}_{8}$, and $\mathrm{La}_{2-x} \mathrm{Sr}_{x} \mathrm{CuO}_{4}$. Squares are the $\mu \mathrm{SR}$ data obtained in the present study. Circles are bulk $\mu$ SR data for $\mathrm{Y}_{1-x} \mathrm{Pr}_{x} \mathrm{Ba}_{2} \mathrm{Cu}_{3} \mathrm{O}_{7-\delta}$ (Refs. 9 and 10) and LE $\mu$ SR data for optimally doped $\mathrm{YBa}_{2} \mathrm{Cu}_{3} \mathrm{O}_{7-\delta}$ (Ref. 11). Triangles are torque magnetization and Meissner fraction data for $\mathrm{La}_{2-x} \mathrm{Sr}_{x} \mathrm{CuO}_{4}$ (Refs. 6 and 8). The lines correspond to the differential Uemura relation with $\Delta \lambda_{a b}(0) / \lambda_{a b}(0)=0.5\left|\Delta T_{c} / T_{c}\right|(A), \Delta \lambda_{a b}(0) / \lambda_{a b}(0)=\left|\Delta T_{c} / T_{c}\right|(B)$, the pseudogap line from Ref. $15(C)$, and the 2D QSI relation given by Eq. (2) $(D)$. The dashed lines indicates that the differential Uemura (line $A$ ) and 2D-QSI (line $D$ ) relations are strictly valid only in the underdoped regime (see text for details).
$=0.5\left|\Delta n_{s} / n_{s}\right|$ (Uemura relation). However, in case (ii) the isotope substitution would lead to a change in $n_{s}$ as well as in $m_{a b}^{*}\left({ }^{16} m_{a b}^{*}<{ }^{18} m_{a b}^{*}\right)$ in order to account for the factor of 2 .

Now we discuss the observed $\Delta \lambda_{a b}(0) / \lambda_{a b}(0)$ vs $\Delta T_{c} / T_{c}$ dependence presented in Fig. 4 in more detail. Tallon et al. ${ }^{15}$ showed that the relation between the oxygen isotope shifts of $\lambda_{a b}(0)$ and $T_{c}$ may be understand in terms of a normal-state pseudogap which competes with superconductivity. Both $\Delta \lambda_{a b}(0) / \lambda_{a b}(0)$ and $\left|\Delta T_{c} / T_{c}\right|$ were found to increase with increasing pseudogap energy $E_{g}$. At the critical doping (when the pseudogap is closed) $\Delta \lambda_{a b}(0) / \lambda_{a b}(0)$ is equal to zero, while $\left|\Delta T_{c} / T_{c}\right| \simeq 0.8 \%$ (line $C$ in Fig. 4). This is, however, inconsistent with the experimental data presented in Fig. 4, especially close to optimal doping (see Fig. 4). Schneider and Keller ${ }^{29-32}$ showed that the relation between the isotope shifts of $\lambda_{a b}(0)$ and $T_{c}$ arises naturally from the dopingdriven three-dimensional to two-dimensional (3D-2D) crossover and 2D quantum superconductor to insulator (2D QSI) transition in the highly underdoped limit. Close to the 2D QSI transition the following relation holds: ${ }^{31,32}$

$$
\Delta \lambda_{a b}(0) / \lambda_{a b}(0)=\left(\Delta d_{s} / d_{s}-\Delta T_{c} / T_{c}\right) / 2,
$$

where $\Delta d_{s} / d_{s}$ is the oxygen isotope shift of the thickness of the superconducting sheets $d_{s}$ of the sample. The best fit of Eq. (2) to the experimental data gives $\Delta d_{s} / d_{s}=3.3(4) \%$ (line $D$ in Fig. 4). Note that Eq. (2) is strictly valid only in the underdoped region. The fit, however, describes the behavior at all doping levels reasonably well. Since the lattice parameters are not modified by oxygen substitution ${ }^{33,34}$ the observation of an isotope effect on $d_{s}$ implies local lattice distortions involving oxygen that are coupled to the superfluid. It was shown that in anisotropic superconductors falling into the 2D XY QSI universality class at zero temperature, the isotope effects on the transition temperature, specific heat, and magnetic field penetration depth are related by a universal relation. This implies a dominant role of fluctuations so that pair formation and pair condensation do not occur simultaneously. From these results Schneider and Keller conclude that the observed isotope effects do not provide direct information on the underlying pairing mechanism and must be attributed to the shift of the phase diagram upon isotope substitution caused by electron-lattice interaction. ${ }^{29-32}$ Bussmann-Holder et al. ${ }^{35-37}$ investigated the origin of the isotope effects on the superconducting transition temperature and the magnetic penetration depth within a polaronic model. For this purpose the well-known $t$ - $J$ Hamiltonian was extended to incorporate the hole induced charge channel and the important effects from the lattice. This results in a twocomponent Hamiltonian, where interactions between the charge channel (local hole plus induced lattice distortion) and the spin channel (antiferromagnetic fluctuations modified by lattice distortions) are explicitly included. ${ }^{35-37}$ This polaronic model predicts for the OIE on $T_{c}$ and $\lambda_{a b}(0)$ the relation $\Delta \lambda_{a b}(0) / \lambda_{a b}(0)=\left|\Delta T_{c} / T_{c}\right|$ (line $D$ in Fig. 4), in agreement with experiments in the underdoped regime.

\section{CONCLUSION}

In conclusion, the oxygen isotope ${ }^{16} \mathrm{O}-{ }^{18} \mathrm{O}$ effects on the in-plane magnetic penetration depth $\lambda_{a b}(0)$ and transition 
temperature $T_{c}$ were studied in optimally doped $\mathrm{YBa}_{2} \mathrm{Cu}_{3} \mathrm{O}_{7-\delta}$ and $\mathrm{La}_{1.85} \mathrm{Sr}_{0.15} \mathrm{CuO}_{4}$, and in the slightly underdoped $\mathrm{YBa}_{2} \mathrm{Cu}_{4} \mathrm{O}_{8}$ and $\mathrm{Y}_{0.8} \mathrm{Pr}_{0.2} \mathrm{Ba}_{2} \mathrm{Cu}_{3} \mathrm{O}_{7-\delta}$ by means of muon-spin rotation and magnetization techniques. A small OIE on the transition temperature $T_{c}$ was observed that is associated with a substantial OIE on the in-plane penetration depth $\lambda_{a b}(0)$, as shown in Fig. 4 and Tables I and II. This finding suggests that lattice effects are directly or indirectly involved in determining the superconducting state. It is worth to note that in colossal magnetoresistance (CMR) manganites similar peculiar OIE on various quantities (e.g., ferromagnetic transition temperature, charge-ordering temperature) were observed, ${ }^{38}$ indicating that in both classes of perovskites, HTSs and CMR manganites, lattice vibrations play an essential role.

\section{ACKNOWLEDGMENTS}

This work was partly performed at the Swiss Muon Source $(\mathrm{S} \mu \mathrm{S})$, Paul Scherrer Institute (PSI, Switzerland). The authors are grateful to D. Di Castro, D. G. Eshchenko, A. Amato, and D. Herlach for assistance during the $\mu \mathrm{SR}$ measurements. This work was supported by the Swiss National Science Foundation, in part by the NCCR program MaNEP, and by the K. Alex Müller Foundation.
${ }^{1}$ B. Batlogg, G. Kourouklis, W. Weber, R. J. Cava, A. Jayaraman, A. E. White, K. T. Short, L. W. Rupp, and E. A. Rietman, Phys. Rev. Lett. 59, 912 (1987).

${ }^{2}$ J. P. Franck, J. Jung, M. A-K. Mohamed, S. Gygax, and G. I. Sproule, Phys. Rev. B 44, 5318 (1991).

${ }^{3}$ J. P. Franck, in Physical Properties of High Temperature Superconductors $I V$, edited by D. M. Ginsberg (World Scientific, Singapore, 1994), pp. 189-293.

${ }^{4}$ D. Zech, H. Keller, K. Conder, E. Kaldis, E. Liarokapis, N. Poulakis, and K. A. Müller, Nature (London) 371, 681 (1994).

${ }^{5}$ G. M. Zhao and D. E. Morris, Phys. Rev. B 51, 16487 (1995).

${ }^{6}$ G. M. Zhao, M. B. Hunt, H. Keller, and K. A. Müller, Nature (London) 385, 236 (1997).

${ }^{7}$ G. M. Zhao, K. Conder, H. Keller, and K. A. Müller, J. Phys.: Condens. Matter 10, 9055 (1998).

${ }^{8}$ J. Hofer, K. Conder, T. Sasagawa, G. M. Zhao, M. Willemin, H. Keller, and K. Kishio, Phys. Rev. Lett. 84, 4192 (2000).

${ }^{9}$ R. Khasanov, A. Shengelaya, K. Conder, E. Morenzoni, I. M. Savić, and H. Keller, J. Phys.: Condens. Matter 15, L17 (2003).

${ }^{10}$ R. Khasanov, A. Shengelaya, E. Morenzoni, M. Angst, K. Conder, I. M. Savić, D. Lampakis, E. Liarokapis, A. Tatsi, and H. Keller, Phys. Rev. B 68, 220506(R) (2003).

${ }^{11}$ R. Khasanov, D. G. Eshchenko, H. Luetkens, E. Morenzoni, T. Prokscha, A. Suter, N. Garifianov, M. Mali, J. Roos, K. Conder, and H. Keller, Phys. Rev. Lett. 92, 057602 (2004).

${ }^{12}$ R. Khasanov, A. Shengelaya, E. Morenzoni, K. Conder, I. M. Savic, and H. Keller, J. Phys.: Condens. Matter 16, S4439 (2004).

${ }^{13}$ H. Keller, Physica B 326, 283 (2003).

${ }^{14} \mathrm{H}$. Keller, in Superconductivity in Complex Systems, edited by K. A. Müller and A. Bussmann-Holder (Springer, Berlin, 2005), p. 143.

${ }^{15}$ J. L. Tallon, R. S. Islam, J. Storey, G. V. M. Williams, and J. R. Cooper, Phys. Rev. Lett. 94, 237002 (2005).

${ }^{16}$ K. Conder, Mater. Sci. Eng., R. 32, 41 (2001).

${ }^{17}$ J. Karpinski, S. Rusiecki, E. Kaldis, B. Bucher, and E. Jilek, Physica C 160, 449 (1989).

${ }^{18}$ P. Zimmermann, H. Keller, S. L. Lee, I. M. Savic, M. Warden, D. Zech, R. Cubitt, E. M. Forgan, E. Kaldis, J. Karpinski, and C. Krüger, Phys. Rev. B 52, 541 (1995).

${ }^{19}$ C. L. Seaman, J. J. Neumeier, M. B. Maple, L. P. Le, G. M. Luke, B. J. Sternlieb, Y. J. Uemura, J. H. Brewer, R. Kadono, R. F.
Kiefl, S. R. Krietzman, and T. M. Riseman, Phys. Rev. B 42, 6801 (1990).

${ }^{20}$ G. Aeppli, E. J. Ansaldo, J. H. Brewer, R. J. Cava, R. F. Kiefl, S. R. Kreitzman, G. M. Luke, and D. R. Noakes, Phys. Rev. B 35, 7129 (1987).

${ }^{21}$ B. Bucher, J. Karpinski, E. Kaldis, and P. Wachter, Physica C 157, 478 (1989).

${ }^{22}$ J. L. Tallon, J. W. Loram, J. R. Cooper, C. Panagopoulos, and C. Bernhard, Phys. Rev. B 68, 180501(R) (2003).

${ }^{23}$ Y. J. Uemura, G. M. Luke, B. J. Sternlieb, J. H. Brewer, J. F. Carolan, W. N. Hardy, R. Kadono, J. R. Kempton, R. F. Kiefl, S. R. Kreitzman, P. Mulhern, T. M. Riseman, D. Ll. Williams, B. X. Yang, S. Uchida, H. Takagi, J. Gopalakrishnan, A. W. Sleight, M. A. Subramanian, C. L. Chien, M. Z. Cieplak, Gang Xiao, V. Y. Lee, B. W. Statt, C. E. Stronach, W. J. Kossler, and X. H. Yu, Phys. Rev. Lett. 62, 2317 (1989).

${ }^{24}$ Y. J. Uemura, L. P. Le, G. M. Luke, B. J. Sternlieb, W. D. Wu, J. H. Brewer, T. M. Riseman, C. L. Seaman, M. B. Maple, M. Ishikawa, D. G. Hinks, J. D. Jorgensen, G. Saito, and H. Yamochi, Phys. Rev. Lett. 66, 2665 (1991).

${ }^{25}$ A. Rüfenacht, J.-P. Locquet, J. Fompeyrine, D. Caimi, and P. Martinoli, Phys. Rev. Lett. 96, 227002 (2006).

${ }^{26}$ J. L. Tallon, C. Bernhard, U. Binninger, A. Hofer, G. V. M. Williams, E. J. Ansaldo, J. I. Budnick, and Ch. Niedermayer, Phys. Rev. Lett. 74, 1008 (1995).

${ }^{27}$ C. Bernhard, Ch. Niedermayer, U. Binninger, A. Hofer, Ch. Wenger, J. L. Tallon, G. V. M. Williams, E. J. Ansaldo, J. I. Budnick, J. I. Stronach, D. R. Noakes, and M. A. BlanksonMills, Phys. Rev. B 52, 10488 (1995).

${ }^{28}$ Ch. Niedermayer, C. Bernhard, U. Binninger, H. Glückler, J. L. Tallon, E. J. Ansaldo, and J. I. Budnick, Phys. Rev. Lett. 71, 1764 (1993).

${ }^{29}$ T. Schneider and H. Keller, Phys. Rev. Lett. 86, 4899 (2001).

${ }^{30}$ T. Schneider, Phys. Rev. B 67, 134514 (2003).

${ }^{31} \mathrm{H}$. Keller and T. Schneider, cond-mat/0401505 (unpublished).

${ }^{32}$ T. Schneider and H. Keller, New J. Phys. 4, 144 (2004).

${ }^{33}$ K. Conder, D. Zech, Ch. Krüger, E. Kaldis, H. Keller, A. W. Hewat, and E. Jilek, in Phase Separation in Cuprate Superconductors, edited by E. Sigmund and K. A. Müller (Springer, Berlin, 1994), p. 210.

${ }^{34}$ F. Raffa, T. Ohno, M. Mali, J. Roos, D. Brinkmann, K. Conder, and M. Eremin, Phys. Rev. Lett. 81, 5912 (1998). 
${ }^{35}$ A. Bussmann-Holder and H. Keller, Eur. Phys. J. B 44, 487 (2005).

${ }^{36}$ A. Bussmann-Holder, H. Keller, and K. A. Müller, in Superconductivity in Complex Systems, edited by K. A. Müller and A. Bussmann-Holder (Springer, Berlin, 2005), p. 365.
${ }^{37}$ A. Bussmann-Holder, H. Keller, A. R. Bishop, A. Simon, R. Micnas, and K. A. Müller, Europhys. Lett. 72, 423 (2005).

${ }^{38}$ G. M. Zhao, H. Keller, R. L. Greene, and K. A. Müller, in Physics of Manganites, edited by T. A. Kaplan and S. D. Mahanti (Kluwer Academic/Plenum Publishers, New York, 1999), p. 221. 